\section{Терапия делириозного}

\section{синдрома у пациентов}

кардиохирургического профиля

в раннем послеоперационном

\section{периоде}

\author{
В.Е. Рубинчик, И.Ю. Кашерининов, А.Е. Баутин, \\ В.А. Мазурок
}

ФГБУ «Национальный медицинский исследовательский центр им. В.А. Алмазова» МЗ РФ, Санкт-Петербург, Россия

\section{Реферат}

Актуальность. Делириозный синдром весьма актуален у пожилых пациентов, переносящих тяжелые операции с применением искусственного кровообращения и проводящих в отделениях реанимации и интенсивной терапии (ОРИТ) достаточно длительное время, лиц, страдающих распространенным атеросклерозом и имеющих широкий спектр сопутствующей патологии.

В мировой литературе встречается множество данных об успешном применении дексмедетомидина с целью седации, предупреждения и купирования делириозного синдрома, в том числе и у пациентов после вмешательств на открытом сердце. Сравнительная оценка медикаментозной седации дексмедетомидином в сочетании с применением нейролептиков и традиционных для РФ методик ведения кардиохирургических пациентов с послеоперационным делирием представляется актуальной клинической и исследовательской задачей.

Цель исследования. Сравнительная оценка эффективности медикаментозной седации дексмедетомидином и пропофолом у пациентов с делириозным синдромом в раннем послеоперационном периоде вмешательств на открытом сердце.

Материалы и методы. В 2017-2019 гг. в ретроспективное описательное исследование включили 42 пациента, перенесших вмешательства на открытом сердце в условиях искусственного кровообращения и имевших в послеоперационном периоде делириозный синдром.

Критерии исключения: стенозирующий атеросклероз брахиоцефальных артерий; острый период или последствия острого нарушения мозгового кровообращения; признаки острой дыхательной недостаточности, связанной с патологией органов системы внешнего дыхания; тяжелая сердечно-сосудистая недостаточность; возраст $>80$ лет.

Выявление и терапия делириозного синдрома производились врачом анестезиологом-реаниматологом совместно с психиатром. В качестве основного нейро-

\section{Treatment of delirium in early postoperative cardiosurgical patients. Article}

\author{
V.E. Rubinchik, I.Yu. Kasherininov, A.E. Bautin \\ Almazov National Medical Research Centre, St. Petersburg, Russia
}

\section{Abstract}

Background. Postoperative delirium is highly relevant in elderly patients who undergo cardiosurgical interventions with cardiopulmonary bypass (CPB), long stay in intensive care unit (ICU), suffering from widespread atherosclerosis and having a wide range of comorbidities.

In the world literature there is a lot of data on the successful use of dexmedetomidine for sedation, prevention and relief of delirium, including patients after open-heart interventions. Comparative evaluation of dexmedetomidine and neuroleptic sedation with "traditional" for Russian Federation management of cardiosurgical patients suffering from postoperative delirium seems to be a relevant clinical and research task.

Objectives. Comparative evaluation of the effectiveness of drug sedation with dexmedetomidine and propofol in cardiosurgical patients with postoperative delirium.

Material and Methods. An open prospective cohort study took place in 2017-2019 and included 42 patients who underwent open-heart intervention with CPB and had delirium in the postoperative period.

Exclusion criteria: severe brachiocephal artery atherosclerosis, acute period or consequences of stroke, signs of acute respiratory failure associated with the pathology of the respiratory system, severe cardiovascular failure, age over 80 years.

Detection and treatment of delirium were performed by an intensivist together with a psychiatrist. Haloperidol was used as the main antipsychotic. Depending on the drug used for medical sedation, the patients were divided into 2 groups: 22 patients underwent medical sedation with propofol, 20 with dexmedetomidine in the form of an extended infusion. According to the results of the study, the length of patients' stay in the ICU and hospital, the need for vasopressor therapy, mechnical ventilation (MV), and the duration of delirium were analyzed.

Results. In patients of the dexmedetomidine group, compared with the propofol group, the following was observed: less need for vasopressors and the duration of their use, less frequent use of $\mathrm{MV}(27.2 \%$ versus $10.0 \%$ of cases, $P<0.05)$, shorter stay in ICU $(4.4 \pm 1.8$ vs $7.2 \pm 2.3$ days, $p<0.05)$, and finally, more rapid relief of the delirium itself $(34.7 \pm 6.8$ vs $52.6 \pm 8.9$ hours, $p<0.05)$. There was 
лептика использовался галоперидол. В зависимости от препарата, используемого для медикаментозной седации, пациенты были разделены на 2 группы: 22 пациентам проводилась медикаментозная седация пропофолом, 20 - дексмедетомидином в виде продленной инфузии. Были проанализированы продолжительность пребывания пациентов в ОРИТ и стационаре, потребность в применении вазопрессоров, потребность в механической респираторной поддержке (МРП) и ее длительность, продолжительность делириозного синдрома.

Результаты. У пациентов из группы дексмедетомидина по сравнению с группой пропофола наблюдались: меньшая потребность в вазопрессорах и длительность их применения, меньшая частота использования МРП (5,0 vs 31,8\% случаев, $p<0,05)$, меньшая длительность пребывания в ОРИТ $(4,4 \pm 1,8$ vs 7,2 $\pm 2,3$ сут, $p<0,05)$, и наконец, более быстрое купирование непосредственно делириозного синдрома (34,7 \pm 6,8 vs 52,6 \pm 8,9 4, $p<0,05)$. По длительности пребывания в стационаре статистически значимого различия между группами получено не было.

Заключение. В раннем послеоперационном периоде вмешательств на открытом сердце медикаментозная седация с применением дексмедетомидина в качестве компонента терапии делириозного синдрома представляется предпочтительной перед седацией с использованием пропофола. Использование дексмедетомидина в рамках проведенного исследования было ассоциировано с более быстрым купированием делириозного синдрома, более благоприятным гемодинамическим профилем, меньшей потребностью в МРП и длительностью искусственной вентияции легких (ИВЛ), а также меньшей длительностью пребывания в ОРИТ в сравнении с использованием медикаментозной седации с применением пропофола, что позволяет рекомендовать ее рутинное использование в ОРИТ кардиохирургического профиля.

Ключевые слова: кардиохирургия, послеоперационный период, делириозный синдром, медикаментозная седация, дексмедетомидин

\ Для корреспонденции: Рубинчик Вадим Ефимович канд. мед. наук, доцент, заведующий отделением анестезиологии-реанимации № 2 ФГБУ «НМИЦ им. В.А. Алмазова» МЗ РФ, Санкт-Петербург; e-mail: verubinchik@gmail. com

๔) Для цитирования: Рубинчик В.Е., Кашерининов И.Ю., Баутин А.Е., Мазурок В.А. Терапия делириозного синдрома у пациентов кардиохирургического профиля в раннем послеоперационном периоде. Вестник интенсивной терапии имени А.И. Салтанова. 2019;3:77-83.

๘ Поступила: 26.03.2019

目 Принятак кечати: 03.09.2019 no statistically significant difference between the groups for the length of hospital stay.

Conclusions. In the early postoperative period of openheart interventions, medical sedation using dexmed etomidine as a component of the delirium therapy seems to be preferable to propofol sedation. Using of dexmedetomidine during study was associated with more rapid relief of the delirium, more favorable hemodynamic profile, less need for MV and duration of $\mathrm{MV}$, as well as shorter duration of stay in the ICU, compared with the use of propofol sedation, which allows us to recommend routine use of dexmedetomidine in the cardiosurgical ICU.

Keywords: cardiosurgery, postoperative period, delirium, sedation, dexmedetomidine

$\otimes$ For correspondence: Vadim E. Rubinchik - M. D., associate professor, the chief of the ICU\#2 of Almazov National Medical Research Centre, St. Petersburg; e-mail: verubinchik@gmail. com

(4) For citation: Rubinchik VE, Kasherininov IYu, Bautin AE. Treatment of delirium in early postoperative cardiosurgical patients. Article. Annals of Critical Care. 2019;3:77-83

c Received: 26.03 .2019

․ A Accepted: 03.09.2019 
Послеоперационное ведение кардиохирургических пациентов представляет собой постоянно совершенствующуюся область интенсивной терапии. Наряду с многообразием факторов риска развития сердечно-сосудистой и дыхательной недостаточности особое внимание в послеоперационном периоде вмешательств на открытом сердце уделяется органическим и функциональным расстройствам центральной нервной системы [1-3]. Делириозный синдром весьма актуален у пожилых пациентов, переносящих тяжелые операции с применением искусственного кровообращения и проводящих в отделении реанимации и интенсивной терапии (ОРИТ) достаточно длительное время, лиц, страдающих распространенным атеросклерозом и имеющих широкий спектр сопутствующей патологии [4-10].

Дексмедетомидин, высокоселективный агонист a-адренорецепторов центрального действия, является препаратом, недавно получившим широкое применение за рубежом и в ряде клиник РФ. Клинический эффект дексмедетомидина в виде обеспечения седации, анальгезии и купирования психомоторного возбуждения был подтвержден рядом крупных исследований [11-21]. В то же время описано и его побочное действие - брадикардия и гипотензия, что требует применения гемодинамического мониторинга во время его использования. Рядом авторов отмечаются такие благоприятные особенности седации дексмедетомидином, как возможность коммуникации с медицинским персоналом [11, 12], снижение выраженности болевого синдрома, тошноты, уменьшение необходимости применения других седативных и анальгетических препаратов, снижение длительности механической респираторной поддержки (МРП) и периода пребывания в ОРИТ [13-20]. В мировой и отечественной литературе встречаются доказательства успешного применения данного препарата у кардиохирургических пациентов [18, 21-26]. Учитывая вышеизложенное, сравнительная оценка медикаментозной седации дексмедетомидином в сочетании с применением умеренных доз нейролептиков и традиционных для РФ методик ведения кардиохирургических пациентов с послеоперационным делирием, включающих в себя использование высоких доз нейролептиков, а также таких гипнотиков, как пропофол и тиопентал натрия, является актуальной клинической и исследовательской задачей.

Цель исследования: сравнительная оценка эффективности медикаментозной седации дексмедетомидином и пропофолом у пациентов с делириозным синдромом в раннем послеоперационном периоде вмешательств на открытом сердце.

\section{Материалы и методы}

Выполнено ретроспективное описательное исследование случаев развития послеоперационного дели- риозного синдрома у пациентов, перенесших вмешательства на открытом сердце в ФГБУ «НМИЦ им. В.А. Алмазова» МЗ РФ в период 2017-2019 гг. Поскольку исследование имело описательный ретроспективный характер, для его проведения не требовалось разрешения локального этического комитета. В исследование были включены пациенты с наличием в раннем послеоперационном периоде делириозного синдрома, о чем судили на основании появления продуктивной симптоматики, психомоторного возбуждения, дезориентации, что подтверждалось консультативным заключением психиатра.

Критериями исключения послужили: наличие у пациентов атеросклероза брахиоцефальных артерий с формированием гемодинамически значимых стенозов; острый период или последствия острого нарушения мозгового кровообращения; признаки острой дыхательной недостаточности, связанной с патологией органов системы внешнего дыхания; тяжелая сердечно-сосудистая недостаточность (использование инотропов/вазопрессоров в дозах, превышающих умеренные: эпинефрин - > 0,05 мкг/кг/мин, норэпинефрин $>0,5$ мкг/кг/мин, дофамин и добутамин - > 5 мкг/кг/ мин); применение устройств вспомогательного кровообращения; возраст > 80 лет.

В исследование включили 29 мужчин и 13 женщин, средний возраст пациентов составил 62,4 \pm 8,9 года. В 26 случаях (61,9\%) пациентам выполнялось изолированное коронарное шунтирование (КШ), в 11 случаях $(26,2 \%)$ - сочетание КШ с пластикой или протезированием клапанов сердца (аортального или митрального клапана), в 5 случаях (11,9\%) - протезирование или пластика клапанов сердца без КШ. Средний индекс массы тела пациентов составил

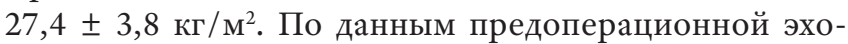
кардиографии, пациенты имели сохранную или умеренно сниженную фракцию выброса левого желудочка > $40 \%$; среднее значение фракции выброса левого желудочка составило 56,8 \pm 8,9\%.

Интраоперационную респираторную поддержку, ингаляционную анестезию и мониторное наблюдение проводили с помощью системы Datex-Ohmeda ADU S/5 (GE Healthcare, СШA). Мониторинг соответствовал локальному протоколу «НМИЦ им. В.А. Алмазова» и международным стандартам для кардиохирургических пациентов. У всех пациентов осуществляли регистрацию 6-канальной ЭКГ, контроль $\mathrm{SpO}_{2}$, инвазивный мониторинг гемодинамики, в том числе методом препульмональной термодилюции с помощью катетера Swan-Ganz. Проводили общую внутривенную либо комбинированную анестезию в условиях искусственной вентиляции легких (ИВЛ) через эндотрахеальную трубку. Анальгезию обеспечивали фентанилом (6 мкг/кг/ч), гипнотический компонент - постоянной инфузией пропофола (6-8 мг/кг/ч) по целевому значению показателя мо- 
нитора энтропии ( $\mathrm{RE}<50 \%)$, миоплегию - пипекурония бромидом (суммарно 0,2-0,25 мг/кг). При использовании комбинированной анестезии на основе севофлурана ингаляционный анестетик подавали в концентрации (ETsev 1,5-2\%), достаточной для поддержания индекса энтропии $<50 \%$, анальгезию осуществляли непрерывной инфузией фентанила (5 мкг/кг ×ч).

Искусственное кровообращение проводили с помощью аппарата Stokert S 3 (Германия): поддерживали среднее перфузионное давление на уровне $70 \pm 5$ мм рт. ст., объемную скорость перфузии - 2,4 л/мин $\times \mathrm{M}^{2}$, нормокапнию. Методика кардиоплегии - изотермическая прерывистая кровяная анте- и ретроградная.

Искусственную вентиляцию легких в послеоперационном периоде проводили в режиме SIMV аппаратами Dräger Savina, Evita 4, Evita XL (Dräger, Германия). B течение первых 3 ч после операции пациенты находились в условиях медикаментозной седации пропофолом и остаточной миоплегии. Послеоперационный мониторинг проводили с применением системы Datex Ohmeda S/5 (GE Healthcare, Финляндия).

Выявление признаков делириозного синдрома производилось врачом анестезиологом-реаниматологом в ходе интенсивной терапии пациентов в условиях ОРИТ, диагноз ставился на основании консультации психиатра. Осмотр пациентов психиатром проводился как на фоне первичного выявления продуктивной симптоматики, так и после купирования делириозного синдрома. Терапия нейролептиками согласовывалась с психиатром на всех этапах ведения пациента. В качестве основного нейролептика использовался галоперидол в виде внутривенного 0,5\% раствора: дробное введение 10-15 мг/сут на фоне медикаментозной седации.

В зависимости от препарата, используемого для медикаментозной седации, ретроспективно пациенты были разделены на 2 группы: 22 пациентам проводилась медикаментозная седация пропофолом (10 мг/ мл), 20 - дексмедетомидином в виде продленной инфузии. Дозировка пропофола подбиралась индивидуально: стартовая доза составляла 4 мг/кг/ч, в дальнейшем проводилось увеличение дозы до необходимой для обеспечения эффективной седации под контролем показателей гемодинамики, оксигенации, газового состава крови. Стартовая доза для дексмедетомидина составляла 0,7 мкг/кг/ч, в последующем производилась коррекция дозировки (в пределах 0,2-1,4 мкг/кг/ч) с целью обеспечения адекватной седации под контролем показателей гемодинамики, оксигенации, газового состава крови.

В течение каждых 12 ч седации инфузия пропофола/дексдора прерывалась с целью оценки уровня сознания и психического статуса пациента. При купировании продуктивной симптоматики, психомоторного возбуждения совместно с психиатром принималось решение о прекращении седации. В случаях сохранения активного делириозного синдрома инфузия препаратов продолжалась до его купирования. В случаях ухудшения параметров оксигенации, газового состава крови на фоне инфузии основного для данной группы препарата проводилась респираторная терапия: ингаляция бронходилататоров и муколитиков через лицевую маску, СРАР-терапия при помощи аппаратов ИВЛ Dräger Evita XL. При неэффективности данных подходов и дальнейшем ухудшении показателей внешнего дыхания пациенты в условиях продолжающейся медикаментозной седации и миоплегии переводились на ИВЛ с продолжением выбранной стратегии лечения делириозного синдрома. По итогам проведенного исследования были проанализированы продолжительность пребывания пациентов в ОРИТ и стационаре, потребность в применении вазопрессоров, МРП, непосредственная продолжительность делириозного синдрома.

Математический анализ выполняли с помощью программы Excel пакета Microsoft Office (Microsoft, США), а также программы Statistica 7.0 (Statsoft Inc., США). Для оценки значимости различий, учитывая ненормальность распределения, применялся непараметрический критерий Вилкоксона. Сравнение качественных показателей проводили с помощью точного критерия Фишера. Изменения считали значимыми при $p<0,05$. Данные представлены в виде медианы (25-й, 75-й процентили).

\section{Результаты исследования}

Исходные данные о пациентах, включенных в исследование, а также характеристика выполненных оперативных вмешательств представлены в табл. 1. Мы не обнаружили каких-либо различий между исследуемыми группами пациентов в приведенных показателях.

Данные о клиническом течении послеоперационного периода для групп пациентов, у которых с целью медикаментозной седации применялись пропофол и дексмедетомидин, представлены в табл. 2.

Данные, представленные в табл. 2, свидетельствуют о том, что у пациентов из группы дексмедетомидина по сравнению с группой пропофола наблюдались: меньшая потребность в вазопрессорах и длительность их применения, меньшая частота и длительность МРП, меньшая длительность пребывания в ОРИТ, и наконец, более быстрое купирование непосредственно делириозного синдрома. По длительности пребывания в стационаре статистически значимого различия между группами получено не было. Один летальный исход в группе пропофола был вызван двусторонней госпитальной пневмонией, сепсисом, острой дыхательной и сердечно-сосудистой недостаточностью. 


\begin{tabular}{|l|c|c|}
\hline \multicolumn{1}{|c|}{ Паблица 1. Характеристика пациентов исследуемых групп $(n=42)$} & Пропофол \\
\hline \multicolumn{1}{|c|}{ Поксмедетомидин } \\
\hline Возраст, лет & $61,8 \pm 8,7$ & $63,0 \pm 9,5$ \\
\hline $\begin{array}{l}\text { Пол: } \\
\text { мужской, } n \text { (\%) }\end{array}$ & $15(68,2 \%)$ \\
женский, $n$ (\%) & $7(31,8 \%)$ & $14(70,0 \%)$ \\
\hline Фракция выброса левого желудочка, \% & $57,1 \pm 8,8$ & $6(30,0 \%)$ \\
\hline Индекс массы тела, кг/м² & $27,3 \pm 3,6$ & $27,5 \pm 4,0$ \\
\hline Площадь поверхности тела, м² & $1,96 \pm 0,17$ \\
\hline Масса тела, кг & $78,9 \pm 7,2$ & $1,95 \pm 0,15$ \\
\hline Характер вмешательств: & & $79,5 \pm 7,5$ \\
КШ & & 12 \\
КШ + протезирование/пластика МК & 3 & 2 \\
КШ протезирование АК & 3 & 3 \\
Протезирование АК & 2 & 3 \\
\hline
\end{tabular}

АК - аортальный клапан; КШ - коронарное шунтирование; МК - митральный клапан.

Таблица 2. Данные о клиническом течении послеоперационного периода у пациентов исследуемых групп (медиана [25-75-й процентили], $n=42)$

\begin{tabular}{|lccc|}
\hline \multicolumn{1}{|c}{ Показатель } & Пропофол $(\boldsymbol{n}=\mathbf{2 2})$ & Дексмедетомидин $(\boldsymbol{n = 2 0 )} \mathbf{P}$ \\
\hline Продолжительность делириозного синдрома, ч & $52,6(47,3-58,4)$ & $34,5(31,3-36,7)$ & $<0,05$ \\
\hline Длительность пребывания в ОРИТ, койко-дни & $7,2(5,7-8,8)$ & $4,4(3,1-5,8)$ & $<0,05$ \\
\hline Длительность госпитализации, койко-дни & $27,6(24,2-30,9)$ & $25,5(22,2-29,0)$ & $>0,05$ \\
\hline Частота переводов на ИВл & $7(31,8 \%)$ & $1(5,0 \%)$ & $<0,05$ \\
\hline Длительность МРП, ч & $58,7(52,1-66,2)$ & $34,5(30,8-38,2)$ & $<0,05$ \\
\hline Потребность в вазопрессорах & $13(59,1 \%)$ & $5(25,0 \%)$ & $<0,05$ \\
\hline Длительность вазопрессорной поддержки, ч & $44,7(37,5-51,9)$ & $28,6(25,4-31,4)$ & $<0,05$ \\
\hline Потребность в галоперидоле, мг/сут & $11,8 \pm 2,2$ & $12,3 \pm 2,6$ & $><0,05$ \\
\hline Летальность & $1(4,5 \%)$ & $>0,05$ \\
\hline
\end{tabular}

ИВЛ - искусственная вентиляция легких; МРП - механическая респираторная поддержка; ОРИТ - отделение реанимации и интенсивной терапии.

\section{Обсуждение}

Результаты проведенного исследования соотносятся с мировыми трендами, иллюстрирующими высокую эффективность и безопасность медикаментозной седации дексмедетомидином в комбинации с нейролептиками для купирования послеоперационного делириозного синдрома. Помимо вышеописанных результатов следует отметить такие преимущества дексмедетомидина, как возможность более эффективного обеспечения энтерального питания (кормление пациентов без установки назогастрального зонда и необходимости парентерального питания), а также улучшение переносимости и увеличение безопасности респираторной терапии.
Использование пропофола у ряда кардиохирургических пациентов ассоциировано со значимым ухудшением гемодинамического профиля, снижением системного артериального давления за счет более выраженного, чем у дексмедетомидина, вазоплегического компонента, что подтвердилось более частым и длительным использованием вазопрессоров в группе пропофола.

Кроме того, пациенты после вмешательств на открытом сердце имеют широкий спектр факторов риска развития острой дыхательной недостаточности, что нередко требует проведения комплексной респираторной терапии. Угнетение сознания и дыхания на фоне инфузии пропофола в ряде случаев потребовало перевода пациентов на ИВЛ, интубации трахеи с целью защиты 
дыхательных путей, что увеличивало время пребывания пациентов в ОРИТ и могло способствовать увеличению риска развития госпитальных пневмоний. Применение дексмедетомидина было ассоциировано со значимо меньшей частотой использования продленной ИВЛ, позволяло сохранять респираторный драйв пациентов и эффективно проводить СРАР-терапию, высокопоточную интраназальную инсуффляцию увлажненного $\mathrm{O}_{2}$, ингаляции бронходилататоров и муколитиков.

В дальнейшем целесообразно проведение дополнительных исследований с оценкой степени тяжести делириозного синдрома, сравнением различных групп антипсихотиков и эффективности их сочетанного применения с дексмедетомидином.

\section{Выводы}

1. В раннем послеоперационном периоде вмешательств на открытом сердце медикаментозная седация с применением дексмедетомидина в качестве компонента терапии делириозного синдрома предпочтительна перед седацией с использованием пропофола.

2. Медикаментозная седация с применением дексмедетомидина у пациентов после операций на открытом сердце ассоциирована с более быстрым купированием делириозного синдрома, более благоприят- ным гемодинамическим профилем, меньшей потребностью в МРП и длительностью ИВЛ, а также меньшей длительностью пребывания в ОРИТ в сравнении с использованием медикаментозной седации с применением пропофола.

3. Высокая эффективность и безопасность медикаментозной седации с применением дексмедетомидина у пациентов с делириозным синдромом в раннем послеоперационном периоде вмешательств на открытом сердце позволяют рекомендовать ее рутинное использование в ОРИТ кардиохирургического профиля.

Конфликт интересов. Авторы заявляют об отсутствии конфликта интересов.

Вклад авторов. Рубинчик В.Е. - разработка плана обзора, систематизация информации и анализ фактических данных, написание и редактирование текста статьи, проверка и утверждение текста статьи; Кашерининов И.Ю., Баутин А.Е., Мазурок В.А. - разработка концепции статьи, получение и анализ фактических данных, написание и редактирование текста статьи, проверка и утверждение текста статьи.

\section{ORCID авторов}

Рубинчик В.Е. - 0000-0003-4501-6420

Кашерининов И.Ю. - 0000-0002-8029-3215

Баутин А.Е. - 0000-0001-5031-7637

Мазурок В.A. - 0000-0003-3917-0771

\section{Литература/References}

[1] McKhann G.M., Grega M.A., Borowicz L.M.Jr., et al. Stroke and encephalopathy after cardiac surgery: an update. Stroke. 2006; 37: 562-571. DOI: 10.1161/01.STR.0000199032.78782.6c

[2] Newman M.F., Grocott H.P., Mathew J.P., et al. Report of the substudy assessing the impact of neurocognitive function on quality of life 5 years after cardiac surgery. Stroke. 2001; 32: 2874-2881.

[3] Selnes O.A., Goldsborough M.A., Borowicz L.M.Jr., et al. Determinants of cognitive change after coronary artery bypass surgery: a multifactorial problem. Ann. Thorac. Surg. 1999; 67: 1669-1676.

[4] Maagaard M., Barbateskovic M., Perner A., et al. Dexmedetomidine for the prevention of delirium in critically ill patients - a protocol for a systematic review. Acta Anaesthesiol. Scand. 2019; 63(4): 540-548. DOI: 10.1111/aas.13313

[5] Collet M.O., CaballeroJ., Sonneville R., et al. Prevalence and risk factors related to haloperidol use for delirium in adult intensive care patients: the multinational AID-ICU inception cohort study. Intensive Care Med. 2018; 44(7): 1081-1089. DOI: 10.1007/s00134018-5204-y

[6] Allen J., Alexander E. Prevention, recognition, and management of delirium in the intensive care unit. AACN Adv. Crit. Care. 2012; 23(1): 5-11; quiz 2-3. DOI: 10.1097/NCl.0b013e31822c3633
[7] Zaal I.J., Slooter A.J. Delirium in critically ill patients: epidemiology, pathophysiology, diagnosis and management. Drugs. 2012; 72(11): 1457-1471. DOI: 10.2165/11635520-000000000-00000

[8] Kalabalik J., Brunetti L., El-Srougy R. Intensive care unit delirium: a review of the literature. J. Pharm. Pract. 2014; 27(2): 195-207. DOI: $10.1177 / 0897190013513804$

[9] Jackson P., Khan A. Delirium in critically ill patients. Crit. Care Clin. 2015; 31(3): 589-603. DOI: 10.1016/j.ccc.2015.03.011

[10] Pandharipande P., Cotton B.A., Shintani A., et al. Prevalence and risk factors for development of delirium in surgical and trauma intensive care unit patients. J. Trauma. 2008; 65(1): 34-41. DOI: 10.1097/TA.0b013e31814b2c4d

[11] Mantz J., Josserand J., Hamada S. Dexmedetomidine: new insights. Eur. J. Anaesthesiol. 2011; 28(1): 3-6. DOI: 10.1097/ EJA.0b013e32833e266d

[12] Gerlach A.T., Dasta J.F. Dexmedetomidine: an updated review. Ann. Pharmacother. 2007; 41: 245-254. DOI: 10.1345/aph.1H314

[13] Honey B.L., Benefield R.J., Miller J.L., Johnson P.N. a2-receptor agonists for treatment and prevention of iatrogenic opioid abstinence syndrome in critically ill patients. Ann Pharmacother. 2009; 43: 1506-1511. DOI: 10.1345/aph.1M161 
[14] Anger K.E. Dexmedetomidine: a review of its use for the management of pain, agitation, and delirium in the intensive care unit. Curr. Pharm. Des. 2013; 19: 4003-4013. DOI: 10.2174/138161281 1319220009

[15] Gerlach A.T., Murphy C.V., Dasta J.F. An updated focused review of dexmedetomidine in adults. Ann. Pharmacother. 2009; 43(12): 2064-2074. DOI: 10.1345/aph.1M310

[16] Keating G.M. Dexmedetomidine: a review of its use for sedation in the intensive care setting. Drugs. 2015; 75(10): 1119-1130. DOI: 10.1007/s40265-015-0419-5

[17] Man Y., Guo Z., Cao J., Mi W. Efficacy of perioperative dexmedetomidine in postoperative neurocognitive function: a meta-analysis. Clin. Exp. Pharmacol. Physiol. 2015; 42(8): 837-842. DOI: 10.1111/1440-1681.12432

[18] Nguyen J., Nacpil N. Effectiveness of dexmedetomidine versus propofol on extubation times, length of stay and mortality rates in adult cardiac surgery patients: a systematic review and meta-analysis. JBI Database System. Rev. Implement. Rep. 2018; 16(5): 1220-1239. DOI: 10.11124/JBISRIR-2017-003488

[19] Peng K., Zhang J., Meng X.W., et al. Optimization of postoperative intravenous patient-controlled analgesia with opioid-dexmedetomidine combinations: an updated meta-analysis with trial sequential analysis of randomized controlled trials. Pain Physician. 2017; 20(7): 569-596.

[20] Zhou C., Zhu Y., Liu Z., Ruan L. Effect of dexmedetomidine on postoperative cognitive dysfunction in elderly patients after general anaesthesia: a meta-analysis. J. Int. Med. Res. 2016; 44(6): 11821190. DOI: $10.1177 / 0300060516671623$

[21] Liu X., Xie G., Zhang K., et al. Dexmedetomidine vs propofol sedation reduces delirium in patients after cardiac surgery: a meta-analysis with trial sequential analysis of randomized controlled trials. J. Crit. Care. 2017; 38: 190-196. DOI: 10.1016/j.jcrc.2016.10.026

[22] Еременко А.А., Чернова Е.В. Применение дексмедетомидина для внутривенной седации и лечения делирия в раннем послеоперационном периоде у кардиохирургических больных. Анестезиология и реаниматология. 2013; 5: 4-8.

[Eremenko A.A., Chernova E.V. Dexomedetomidine use for intravenous sedation and delirium treatment during early postoper- ative period in cardio-surgical patients. Anesteziologia i reanimatologia. 2013; 5: 4-8. (In Russ)]

[23] Еременко А.А., Чернова Е.В. Лечение делирия в раннем послеоперационном периоде у кардиохирургических пациентов. Анестезиология и реаниматология. 2014; 3: 30-34.

[Eremenko A.A., Chernova E.V. Treatment of delirium in cardio-surgical patients in early postoperative period. Anesteziologia i reanimatologia. 2014; 3: 30-34. (In Russ)]

[24] Еременко А.А., Чернова Е.В. Сравнение дексмедетомидина и пропофола при внутривенной седации в раннем послеоперационном периоде у кардиохирургических пациентов. Анестезиология и реаниматология. 2014; 2: 37-41.

[Eremenko A.A., Chernova E.V. Comparison of dexmedetomidine and propofol for short-term sedation in early postoperative period after cardiac surgery. Anesteziologia i reanimatologia. 2014; 2: 37-41. (In Russ)]

[25] Никода В.В., Грицан А.И., Еременко А.А., et al. Эффективность и безопасность применения дексмедетомидина для седации больных при проведении продленной ИВЛ в отделениях реанимации и интенсивной терапии (результаты российского многоуровневого исследования). Анестезиология и реаниматология. 2015; 60(5): 47-53.

[Nikoda V.V., Gritsan A.I., Eremenko A.A., et al. The efficacy and safety of dexmedetomidine for sedation of patients during prolonged mechanical ventilation in intensive care units (Russian multicenter study results). Anesteziologia i reanimatologia. 2015; 60(5): 47-53. (In Russ)]

[26] Линев Д.В., Ярошецкий А.И., Проценко Д.Н., Гельфанд Б.Р. Эффективность и безопасность дексмедетомидина, галоперидола и диазепама в лечении делирия: сравнительное исследование. Анестезиология и реаниматология. 2017; 62(6): 442-448. DOI: 10.18821/0201-7563-2017-62-6-442-448

[Linev D.V., Yaroshetskiy A.I., Protsenko D.N., Gel'fand B.R. The efficacy and the safety of dexmedetomidine, haloperidol and diazepam for treatment of delirium: a comparative study. Anesteziologiya i reanimatologiya (Russian Journal of Anaesthesiology and Reanimatology). 2017; 62(6): 442-448. (In Russ)] 